C-A/AP/\#148 April 2004

\title{
Cancellation of the Chromatic Effects with proper Field Profile
}

\author{
Alessandro G. Ruggiero
}

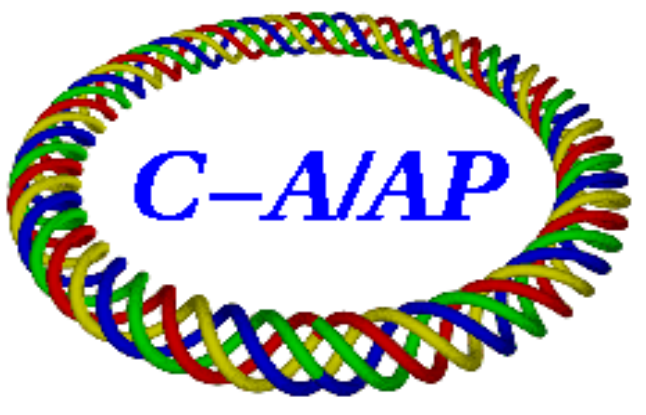

Collider-Accelerator Department Brookhaven National Laboratory Upton, NY 11973 


\title{
Cancellation of the Chromatic Effects with proper Field Profile
}

\author{
(Sandro's Recipe) \\ Alessandro G. Ruggiero \\ Brookhaven National Laboratory \\ March 2004
}

\section{FFAG Configuration}

The AGS-FFAG ring is made of a number of identical periods shown in Figure 1 below. There is a reference trajectory, also shown in the same Figure 1, that corresponds to the central momentum value $\mathrm{p}_{0}$. The reference trajectory is made of arcs of circle in the sector magnets $F$ and $D$ with constant curvature respectively $h_{1}$ and $h_{2}$, and of straight lines in the drift regions $S$ and $g$ with vanishing curvature $h=0$. The opposing exit and entrance planes of the neighboring sector magnets are parallel to each other; thus, the entrance and exit angles of the reference trajectory with each magnet are identically zero.

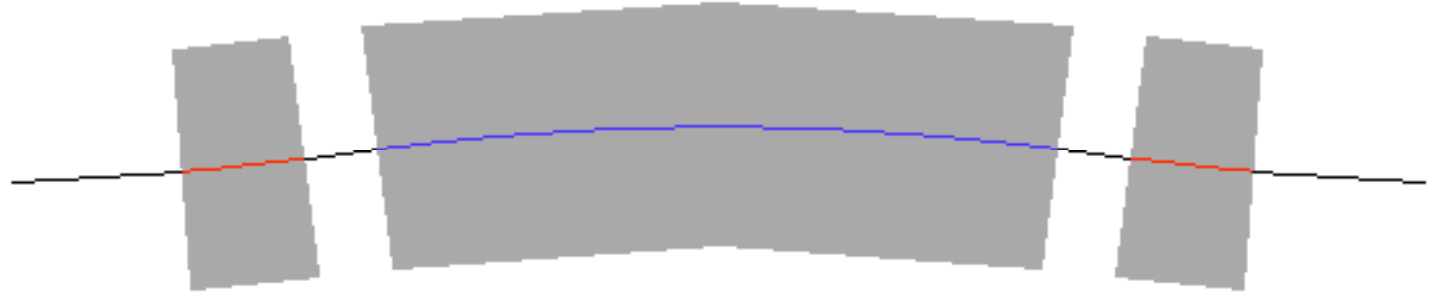

Figure 1. One period structure of the FFAG lattice

\section{Derivation of the Equations of Motion}

We adopt a curvilinear coordinate system ( $\mathrm{x}, \mathrm{s}, \mathrm{y})$ where $\mathrm{s}$ is the path length along the reference trajectory, and $\mathrm{x}, \mathrm{y}$ are respectively the horizontal and vertical displacements from the reference trajectory. Assuming that the motion is in the anticlockwise direction, along the increasing direction of $\mathrm{s}, \mathrm{x}$ has positive value to the outside and $\mathrm{y}$ above the mid-plane.

The equations of motion for any particle with momentum $\mathrm{p}$ and electric charge $\mathrm{q}$ are derived from the (exact) Hamiltonian

$H=-q A_{s} / c-(1+h x)\left[p^{2}-\left(p_{x}-q A_{x} / c\right)^{2}-\left(p_{y}-q A_{y} / c\right)^{2}\right]^{1 / 2}$

where $c$ is the light velocity, $h=h(s)$ the curvature of the reference trajectory, and $p_{x}, p_{y}$ are the transverse components of the particle momentum canonically conjugated to $\mathrm{x}, \mathrm{y}$.

The equations of motion are 


$$
\begin{array}{ll}
\mathrm{p}_{\mathrm{x}}{ }^{\prime}=-\partial \mathrm{H} / \partial \mathrm{x} & \mathrm{x}^{\prime}=\partial \mathrm{H} / \partial \mathrm{p}_{\mathrm{x}} \\
\mathrm{p}_{\mathrm{y}}{ }^{\prime}=-\partial \mathrm{H} / \partial \mathrm{y} & \mathrm{y}^{\prime}=\partial \mathrm{H} / \partial \mathrm{p}_{\mathrm{y}}
\end{array}
$$

with a prime denoting differentiation with respect to $s\left({ }^{\prime} \equiv \mathrm{d} / \mathrm{ds}\right)$. Expanding the square root at the r. h. side of (1) and retaining only up to quadratic order terms give*:

$H=-q A_{s} / c-(1+h x) p+\left(p_{x}-q A_{x} / c\right)^{2} / 2 p+\left(p_{y}-q A_{y} / c\right)^{2} / 2 p$

after having also dropped higher order terms like $\mathrm{hx}\left(\mathrm{p}_{\mathrm{x}}-\mathrm{qA}_{\mathrm{x}} / \mathrm{c}\right)^{2}$ and $\mathrm{hx}\left(\mathrm{p}_{\mathrm{y}}-\mathrm{qA}_{\mathrm{y}} / \mathrm{c}\right)^{2}$.

We assume that the magnetic field distribution in the proximity of the reference trajectory is given solely by the longitudinal component $\mathrm{A}_{\mathrm{s}}$ of the vector potential, whereas identically $A_{x}=A_{y}=0$. In this case the equations of motion become

$$
\begin{aligned}
& \mathrm{x}^{\prime \prime}=(\mathrm{q} / \mathrm{pc}) \partial \mathrm{A}_{\mathrm{s}} / \partial \mathrm{x}+\mathrm{h} \\
& \mathrm{y}^{\prime \prime}=(\mathrm{q} / \mathrm{pc}) \partial \mathrm{A}_{\mathrm{s}} / \partial \mathrm{y}
\end{aligned}
$$

This is correct only as long $A_{s}$ does not vary with the longitudinal position within the length of one magnet; otherwise a solenoid field component is introduced that should be taken into account in the equations of motion.

\section{Magnetic Field Components}

With the chosen curvilinear coordinate system, the components $\mathrm{B}_{\mathrm{x}}$ and $\mathrm{B}_{\mathrm{y}}$ of the magnetic field are $\left(B_{s}=0\right)$

$$
\begin{aligned}
& (1+\mathrm{hx}) \mathrm{B}_{\mathrm{y}}=\partial \mathrm{A}_{\mathrm{s}} / \partial \mathrm{x} \\
& (1+\mathrm{hx}) \mathrm{B}_{\mathrm{x}}=-\partial \mathrm{A}_{\mathrm{s}} / \partial \mathrm{y}
\end{aligned}
$$

and the equations of motion are now

$$
\begin{aligned}
& \mathrm{x}^{\prime \prime}=(\mathrm{q} / \mathrm{pc}) \mathrm{B}_{\mathrm{y}}(1+\mathrm{hx})+\mathrm{h} \\
& \mathrm{y}^{\prime \prime}=-(\mathrm{q} / \mathrm{pc}) \mathrm{B}_{\mathrm{x}}(1+\mathrm{hx})
\end{aligned}
$$

Formally, using complex notation, we can write

$$
\begin{aligned}
& B=B_{y}+i B_{x} \\
& z=x+i y
\end{aligned}
$$

Quite generally

\footnotetext{
${ }^{*}$ This may not be necessary if the equations of motion are to be integrated with MATHEMATICA
} 
$\mathrm{B}(\mathrm{z})=\mathrm{B}_{0}+\mathrm{G}(\mathrm{z}) \mathrm{z}$

where $\mathrm{B}_{0}$ is a constant, that is the bending field on the reference trajectory, and $\mathrm{G}(\mathrm{z})$ includes the field gradient and higher order nonlinearities. We have

$\mathrm{B}_{\mathrm{x}}=\operatorname{Imaginary}\{\mathrm{B}(\mathrm{z})\}$

$\mathrm{B}_{\mathrm{y}}=\operatorname{Real}\{\mathrm{B}(\mathrm{z})\}$

\section{Chromatic Effects on the Horizontal Plane}

Let us consider now the motion on the mid-plane where $\mathrm{y}=0$,

$\mathrm{x}^{\prime \prime}=(\mathrm{q} / \mathrm{pc})\left(\mathrm{B}_{0}+\mathrm{Gx}\right)(1+\mathrm{hx})+\mathrm{h}$

But the bending condition on the reference orbit requires

$\left(\mathrm{qB}_{0} / \mathrm{p}_{0} \mathrm{c}\right)=-\mathrm{h}$

Introduce also the relative momentum deviation $\mathrm{p}=\mathrm{p}_{0}(1+\delta)$. Neglecting the higher order term ${ }^{*}$ (q / pc) G h x , the horizontal equation of motion (11) reduces to

$$
\begin{aligned}
\mathrm{x}^{\prime \prime} & =(\mathrm{q} / \mathrm{pc})\left(\mathrm{hB}_{0}+\mathrm{G}\right) \mathrm{x}+\mathrm{h}+\mathrm{q} \mathrm{B}_{0} / \mathrm{pc} \\
& =-\mathrm{h}^{2}\left(1+\mathrm{G} / \mathrm{hB} \mathrm{B}_{0}\right) \mathrm{x} /(1+\delta)+\mathrm{h} \delta /(1+\delta)
\end{aligned}
$$

or, introducing the field index $\mathrm{n}=\mathrm{G} / \mathrm{h} \mathrm{B}_{0}$

$\mathrm{x}^{\prime \prime}+\mathrm{h}^{2}(1+\mathrm{n}) \mathrm{x} /(1+\delta)=\mathrm{h} \delta /(1+\delta)$

Like $\mathrm{G}$, also the field index $\mathrm{n}$ is in general a function of $\mathrm{x}$ (and $\mathrm{y}$, or $\mathrm{z}$ ). Similarly, in proximity of the mid-plane, the vertical equation of motion is

$\mathrm{y}^{\prime \prime} \quad-\mathrm{h}^{2} \mathrm{ny} /(1+\delta) \quad=\quad 0$

after having dropped a higher order term in hx.

Equations (14 and 15) above can be in general solved numerically. In the special case the field index $\mathrm{n}$ is constant, the solution can be found analytically, and the well-known matrix method can be used to estimate the usual lattice functions. Because of the explicit momentum dependence at the denominator of both terms, the solution exhibits strong dispersive and chromatic effects that one would like to reduce with some external corrective means.

\footnotetext{
* This may not be necessary, since we shall anyway have to solve a non-linear differential equation. In any case, the contribution of that term is small as long $\mathrm{h} \mathrm{x}<<1$.
} 


\section{Formulation of the Problem}

Let us consider the most general case where the field index is a nonlinear function of both $\mathrm{x}$ and $\mathrm{s}$, namely $\mathrm{n}=\mathrm{n}(\mathrm{x}, \mathrm{s})$. We know that, at any location $\mathrm{s}$, for each momentum value $\delta$ there is one and only one unique solution $\mathrm{x}=\mathrm{x}(\delta, \mathrm{s})$, and by inversion, topologically, $\delta$ can be taken as a function of $\mathrm{x}$ and s, namely $\delta=\delta(\mathrm{x}, \mathrm{s})$. We pose the following problem: Determine the field distribution, namely $\mathrm{n}=\mathrm{n}(\mathrm{x}, \mathrm{s})$, that compensates (mostly) exactly the momentum dependence of $(1+\delta)$ at the denominator. We shall write such solution as

$\mathrm{n}(\mathrm{x}, \mathrm{s})=\mathrm{n}_{0}[1+\delta(\mathrm{x}, \mathrm{s})]$

where $\mathrm{n}_{0}$ is related to the gradient $\mathrm{G}_{0}=\mathrm{n}_{0} \mathrm{~h} \mathrm{~B}_{0}$ on the reference trajectory. If such field distribution is found then the equations (14 and 15) of motion reduce simply to

$$
\begin{aligned}
& \mathrm{x}^{\prime \prime}+\mathrm{h}^{2} \mathrm{x} /(1+\delta)+\mathrm{h}^{2} \mathrm{n}_{0} \mathrm{x}=\mathrm{h} \delta /(1+\delta) \\
& \mathrm{y}^{\prime \prime}-\mathrm{h}^{2} \mathrm{n}_{0} \mathrm{y}=0
\end{aligned}
$$

Notice that this approach does not cancel the momentum dependence of the curvature term, nor the constant dispersive term at the $\mathrm{r}$. $\mathrm{h}$. side of the same equation. Thus, only a partial momentum compensation can be expected, though as we shall see very significant.

\section{Integration of the Equation of Motion}

The solution of either equation (14) or (17a) can be written as the sum of two contributions

$\mathrm{x}(\delta, \mathrm{s})=\mathrm{x}_{\mathrm{c}}(\delta, \mathrm{s})+\mathrm{u}(\delta, \mathrm{s})$

where $x_{c}$ is a particular solution of the inhomogeneous equation (14 or 17a) that has a periodic behavior, and $\mathrm{u}$ is the free betatron solution that satisfies the associated homogenous equation. The solution of equation (17a) is straightforward and can be found with the well-known matrix notation. After that, once the closed orbit is found, the solution can be inverted either numerically or symbolically as done by MATHEMATICA. The inversion will provide the dependence of the momentum deviation with the radial displacement at any given location $\mathrm{s}$,

$\delta=\delta(\mathrm{x}, \mathrm{s})$

that we can insert back in the field expression to derive the equation of motion

$\mathrm{x}^{\prime \prime}+\mathrm{h}^{2}\left\{1+\mathrm{n}_{0}[1+\delta(\mathrm{x}, \mathrm{s})]\right\} \mathrm{x} /(1+\delta)=\mathrm{h} \delta /(1+\delta)$

Proceeding backward, this corresponds to the field distribution on the mid-plane given by

$\mathrm{B}(\mathrm{x}, \mathrm{s})=\mathrm{B}_{0}+\mathrm{G}_{0}[1+\delta(\mathrm{x}, \mathrm{s})] \mathrm{x}$ 


\section{Prove of the Validity of the proposed Approach}

Equation (20) combined to (21), though nonlinear, can be integrated. The solution is indeed expected to have removed the chromatic behavior caused by the momentum dependence of the magnetic field. The integration of (20) can be attempted in two possible ways:

(i) Numerical integration by starting with some initial conditions $x_{0}(\delta)$ and $x_{0}{ }^{\prime}(\delta)$ and looking for a solution that closes at the end of the period. To facilitate the search for the closed orbit solution, the initial conditions can be set from the originally determined solution $\mathrm{x}_{\mathrm{c}}(\delta, \mathrm{s})$ of the linear equation $(17 \mathrm{a})$.

(ii) By direct substitution of the originally determined solution $\mathrm{x}_{\mathrm{c}}(\delta, \mathrm{s})$ of the linear equation (17a) into equation (20), and by recording the relative deviation.

Either of these two methods, if successful, will have proven the validity of the Approach.

\section{Alternative Solutions}

The field profile of equation (21), derived according to the recipe specified above, yields a vertical betatron tune entirely independent of the momentum deviation $\delta$, as it is possible to see by inspecting (17b); but there is still a residual dependence of the horizontal tune with $\delta$ due to the curvature term.

Other solutions are of course also possible. For instance, in order to flatten entirely the $\delta$ dependence of the horizontal betatron tune, one can set the field profile so that

$1+\mathrm{n}(\mathrm{x}, \mathrm{s})=\left(1+\mathrm{n}_{0}\right)[1+\delta(\mathrm{x}, \mathrm{s})]$

which yields the following equations of motion

$$
\begin{array}{llll}
\mathrm{x}^{\prime \prime} & +\mathrm{h}^{2}\left(1+\mathrm{n}_{0}\right) \mathrm{x} & =\mathrm{h} \delta /(1+\delta) \\
\mathrm{y}^{\prime \prime} & - & \mathrm{h}^{2}\left[\delta /(1+\delta)+\mathrm{n}_{0}\right] \mathrm{y} & =
\end{array}
$$

There is now a (reduced) $\delta$-dependence of the vertical betatron tune. There is, of course, still dispersion on the horizontal plane.

The field profile on the $y=0$ mid-plane associated to this solution is given by

$\mathrm{B}(\mathrm{x}, \mathrm{s})=\mathrm{B}_{0}+\mathrm{G}_{0}\left[1+\delta(\mathrm{x}, \mathrm{s})\left(1+1 / \mathrm{n}_{0}\right)\right]$

that, as long $\mathrm{n}_{0} \gg>1$, is only slightly different from the field profile of equation (21).

If desired, always in the search for an optimum cancellation of the chromatic and dispersive behavior, intermediate solutions between (16) and (21) can be found. 